\title{
A role for Msx genes in mammalian embryonic diapause
}

\author{
J Cha ${ }^{1,4}$, JC Fenelon², BD Murphy ${ }^{3}$, G Shaw ${ }^{2}$, MB Renfree ${ }^{2}$ and SK Dey ${ }^{1}$ \\ ${ }^{1}$ Division of Reproductive Sciences, Cincinnati Children's Hospital Medical Center, USA \\ ${ }^{2}$ School of Biosciences, University of Melbourne, Melbourne, Australia \\ ${ }^{3}$ Centre de recherche en reproduction et fertilité, Université de Montréal, Québec, \\ Canada \\ ${ }^{4}$ Present address: Division of Diabetes, Endocrinology, and Metabolism, Department of \\ Medicine, Vanderbilt University Medical Center, USA
}

Corresponding author e-mail: jeeyeon.cha@vumc.org

\begin{abstract}
Mammalian embryonic diapause is a reproductive phenomenon defined by the reversible arrest in blastocyst development and metabolic activity within the uterus which synchronously becomes quiescent to implantation. This natural strategy, evident in over 130 species across eight orders, can temporally uncouple conception from delivery until conditions are favorable for the survival of the mother and newborn. While the maternal endocrine milieu has been shown to be important for this process, the local molecular mechanisms by which the uterus and embryo achieve quiescence, maintain blastocyst survival and then resumes blastocyst activation with subsequent implantation in response to endocrine cues remains unclear. Here we review the first evidence that the proximal molecular control of embryonic diapause is conserved in three unrelated mammalian species which employ different endocrine programs to initiate diapause. In particular, uterine expression of muscle segment homeobox (Msx) genes Msx1 or Msx2 persists during diapause, followed by downregulation with blastocyst reactivation and implantation. Mice (Mus musculus) with conditional inactivation of Msx1 and Msx2 in the uterus fail to achieve diapause and reactivation. Remarkably, the mink (Neovison vison) and tammar wallaby (Macropus eugenii) share this pattern of MSX1 or MSX2 expression as in mice during delay - it persists during diapause and is rapidly downregulated upon implantation. Therefore, these findings were the first to provide evidence that there are common conserved molecular regulators in the uterus of unrelated mammals during embryonic diapause.
\end{abstract}

\section{Introduction}

Mammalian embryonic diapause is a maternal reproductive strategy characterized by the temporary arrest of blastocyst development within the uterus which is also synchronously quiescent with blastocyst dormancy. Reactivation is triggered by the coincident maturity of the endometrium to a receptive phase and activation of the blastocyst for subsequent implantation [1-3]. The endocrine cues that initiate reactivation can vary across species, however, they all effectively uncouple implantation from birth until conditions become favourable for neonatal survival.

In mice, embryonic diapause can occur after postpartum mating during lactation induced by sucking pups (termed facultative delay). The blastocyst remains dormant within a quiescent uterus as the newly born pups suck. In mice, this originates from increased pituitary prolactin secretion 
promoted by the sucking stimulus which downregulates ovarian oestrogen secretion $[4,5]$. Removal of pups (i.e. of the sucking stimulus) initiates embryonic reactivation for implantation via the resultant ovarian oestrogen pulse. This lactational diapause in rodents is thought to prevent metabolic stress from nursing overlapping litters [6] (Figure 1).

Diapause and reactivation in mice can also be experimentally controlled via a number of different methods. Surgically-induced diapause can be induced by ovariectomy before the pre-implantation oestrogen (E2) secretion on the morning of day 4 of pregnancy up to several weeks (between 0800 and $0900 \mathrm{~h}$ ) and can be maintained with continued treatment with progesterone (P4) [7-9]. Alternatively, administering an anti-oestrogen compound (i.e. ICI 182,780) on day 3 at $1800 \mathrm{~h}$ and day 4 between 0800 and $0900 \mathrm{~h}$ also induces delay. Blastocyst reactivation for implantation can then be initiated with administration of E2 or its downstream mediator, leukemia inhibitor factor (LIF) [5].

Lactational induction of diapause is also apparent in other diapausing mammals, such as the tammar wallaby (Macropus eugenii; Fenelon et al. this volume). In this species, heightened prolactin is luteostatic and inhibits $\mathrm{P} 4$ secretion, which is required for reactivation for implantation. Removal of the pouch young, i.e. removal of the sucking stimulus, after the summer solstice (December) triggers reactivation. Intriguingly, tammar wallabies also practice a seasonal diapause controlled by photoperiod after the winter solstice (June) [10]. Carnivorous mammals such as the mustelid mink and ferret, among others, similarly have a seasonal (obligate) diapause regulated by prolactin and determined by day length and melatonin secretion with embryonic development halted at the blastocyst stage (Murphy \& Fenelon this volume) [2, 3] (Figure 1).

In all species studied to date, embryonic diapause is largely governed by the maternal endocrine milieu, although the endocrine signature varies across species $[2,11]$ (Figure 1). While it is known that diapause can only occur when uterine quiescence is coordinated with blastocyst dormancy, the molecular programming required to achieve quiescence, promote embryonic survival, then resume implantation when conditions become favorable, remains largely unknown. The first uterine factors to be implicated in mediating this uterine quiescence are the muscle segment homeobox (MSX) genes MSX1 and MSX2 [12].

\section{Msx genes are required to initiate and maintain diapause in mice.}

Msx1 and Msx2 are members of a highly conserved homeobox gene family [13]. Primarily, Msx proteins function as transcriptional repressors and are known to regulate epithelial-mesenchymal signaling in several contexts [14]. Not surprisingly, the Msx family has a number of important roles throughout development, including neuro-ectodermal patterning and differentiation for central nervous system development, craniofacial development, myogenesis and limb development. Both Msx1 and Msx2 show overlapping but distinct patterns, and genetic knockout models of each family member in mice show distinct effects on developmental processes [14]. In the adult mouse, Msx genes are also expressed in the skin, mammary gland and uterine epithelium [14].

In the mouse uterus under diapausing conditions, there is robust Msx1 expression in the luminal and glandular epithelium for the duration of diapause (Figure 2, top panels). Under surgicallyinduced diapausing conditions, Msx is progressively downregulated upon reactivation with E2 or LIF with reduced expression at the implantation sites by $24 \mathrm{~h}$ to undetectable uterine levels by $48 \mathrm{~h}$ [15]. This pattern of Msx1 expression is maintained regardless of the approach to elicit diapause and reactivation (e.g. experimental or natural). In addition, infertile female mice with genetic deletion of Lif show persistent Msx1 expression with recovery of dormant, elongated embryos 
[15]. Together, these findings suggested that Msx1 could be critical to synchronize uterine quiescence and blastocyst dormancy.

Figure 1

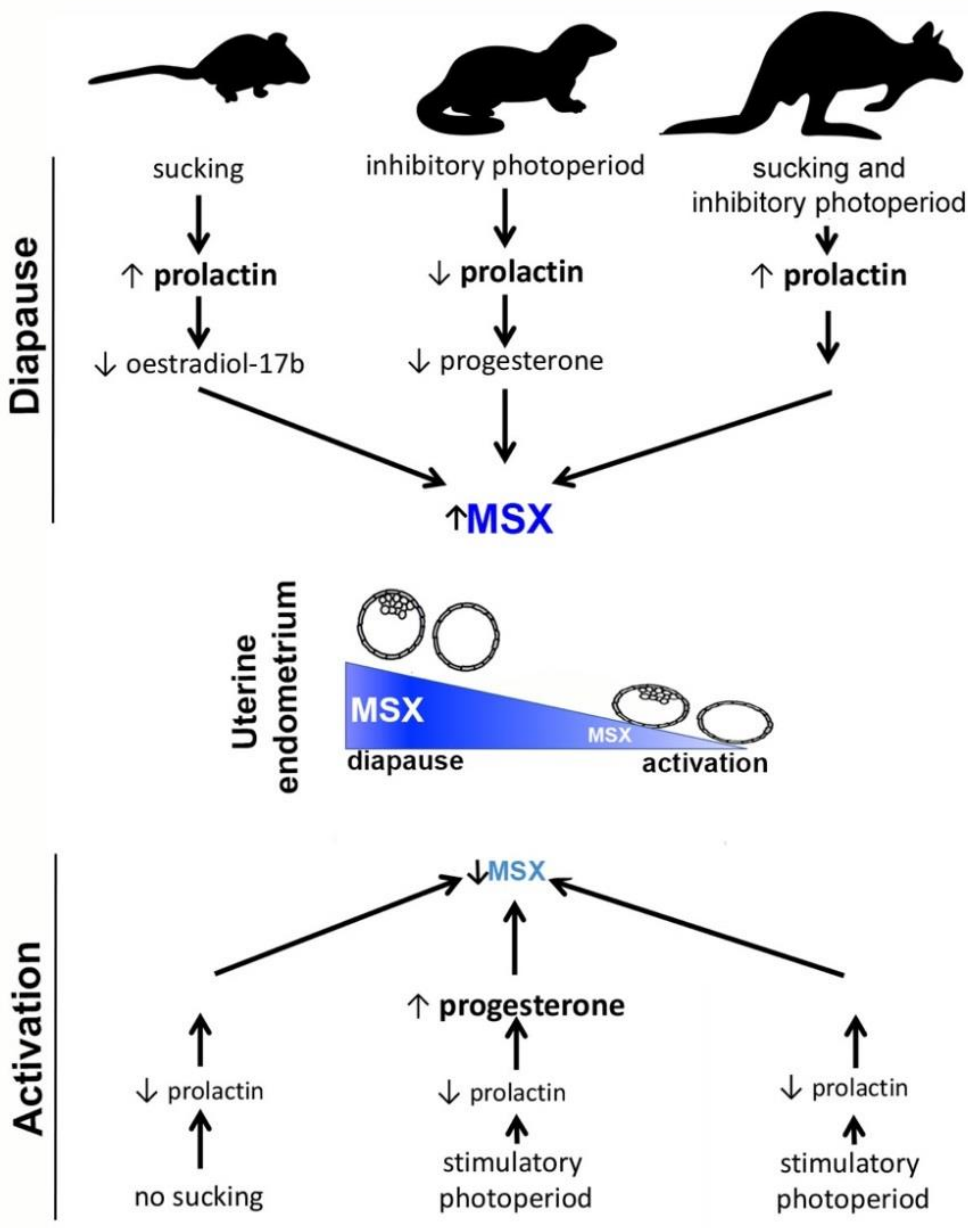

Figure 1. MSX expression persists in diapausing mouse, mink and tammar wallaby uteri.

Although there is variation in the endocrine stimuli used to induce diapause and reactivation in these species in relation to the differing roles of prolactin, progesterone (P4) and oestradiol-17 $\beta$ (E2), these diverse pathways ultimately converge proximally through MSX in the uterus, which controls diapause and reactivation of the blastocyst, with an inner cell mass in eutherian mammals but not in marsupials. Bolded font indicates higher levels of hormones. Blue shade and larger font indicate higher expression of uterine MSX. Blastocyst dormancy is indicated by rounded shape without interaction with the endometrium, while blastocyst activation is indicated by interaction with the endometrium.

Mice with uterine deletion of Msx1 have compromised fertility but are not infertile due to partial compensation of function by uterine Msx2 [15]. However, mice with conditional uterine deletion of both Msx1 and Msx2 (Msx1/Msx2d/d) are infertile due to implantation failure. In the absence of Msx1 and Msx2, required changes in uterine luminal epithelial cell polarity necessary for implantation are compromised, a process mediated via increased Wnt5a signalling [15].

Under diapausing conditions, significantly fewer dormant blastocysts can be recovered in ovariectomized, P4-treated Msx1/Msx2d/d females on day 8 (after 4 days of dormancy) compared with littermate Msx1/Msx2f/f (control) females. Embryos recovered from delayed implanting 
Msx1/Msx2d/d uteri had abnormal morphology and signs of degeneration, suggesting that uteri lacking Msx genes under dormant conditions are not conducive for embryonic survival. When delayed conditions are extended until day 10 of pregnancy (after 6 days of dormancy), the number of recovered blastocysts is further diminished [12].

Intriguingly, a subset of Msx1/Msx2d/d females (33\%) under delayed implanting conditions showed signs suggestive of inappropriate embryonic reactivation and attachment. Some of the recovered blastocysts from these mice have epithelial cells adherent to the embryonic trophectoderm at the abembryonic pole and their locations could be demarcated by faint blue bands upon intravenous injection of a blue dye, suggestive of the intimate apposition and vascular permeability of early implantation. However, these "pseudoimplantation" sites did not result in a pregnancy and ultimately underwent resorption [12].

Expression of MSX in the mink and wallaby during diapause and reactivation is similar to mice.

Remarkably, examination of MSX genes in two other distantly-related diapausing mammals, the mink and the tammar wallaby, indicated that uterine expression of MSX during diapause is conserved [12]. In the mink, reactivation from diapause can be initiated by prolactin administration (day 0, or d0 PRL) [16] (Figure 1). In the diapausing mink uteri, there is high MSX1 expression in both the luminal and glandular epithelial cells with reduced expression after reactivation (d9 PRL) and undetectable levels at implantation sites (d14 PRL) (Figure 2). Notably, compensatory MSX2 expression is not detected [12].

In the tammar wallaby, reactivation requires removal of the pouch young (RPY) (i.e. removal of the sucking stimulus with reduced prolactin secretion) (Figure 1). Although MSX1 is undetectable in the wallaby uterus during diapause, MSX2 under these conditions undergoes progressively reduced expression after blastocyst activation and approaching attachment, which occurs on $\mathrm{d} 18$ RPY, and expression is undetectable prior to birth on d26 RPY (Figure 2). This expression pattern is predominant in the endometrial epithelium, with both MSX2 mRNA and protein being highly expressed in the glandular and surface epithelial during diapause. Notably, upon embryonic reactivation, MSX2 protein expression first clears in the surface epithelium and epithelia of the superficial glands, with deeper glands having intact MSX2 until later stages approaching attachment (undetectable by day 17 after RPY).

\section{What is MSX doing in the uterus during diapause?}

Comparison of the proteomics profiles between $M s \times 1 / M s \times 2^{f / f}$ and $M s \times 1 / M s \times 2^{d / d}$ mouse uteri during diapause identified 448 proteins by $\geq 2$ peptides that are differentially expressed [17]. Network pathway analysis of these proteins identified several interactions between inflammatory

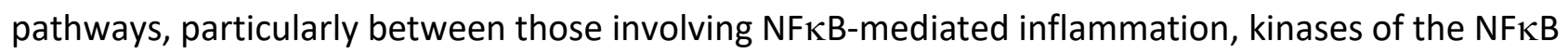
inhibitor IKB (IKK $\beta$ and IKKE), the ubiquitin-proteasome system, endoplasmic reticulum stress, chaperone-mediated protein folding, and oxidative stress [17]. Many of the inflammatory pathways identified by this proteomics and computational analysis intersected on NFKB-mediated inflammation. Examination of this pathway in more detail revealed that there is enhanced $I \kappa B$ phosphorylation during diapause in $M s \times 1 / M s \times 2^{d / d}$ uteri [17]. Phosphorylation of I $\mathrm{B}$ by IKK allows for ubiquitination of $I \kappa B$ and thus targets it for degradation, permitting NFKB to dissociate and translocate to the nucleus to activate transcription of inflammatory mediators. This is supported by increased uterine immunolocalization of nuclear NFKB, particularly in the epithelium and subepithelial stroma of $M s \times 1 / M s \times 2 d / d$ uteri and by the immunolocalization of Cox2 in the luminal epithelium, away from the site of the blastocyst [17]. In sum, these results suggest that the 
increase in inflammation seen in $M s \times 1 / M s \times 2^{d / d}$ uteri under delayed conditions, which prevented a true diapause, are mediated by increased NFKB signaling.

\section{Implications of these findings}

The extraordinary findings of similar patterns of MsX and MSX gene expression in representative species across three mammalian orders using diverse reproductive strategies for diapause has identified a role for these ancient genes in embryonic diapause. Our study in mice (Eutheria: Rodentia), mink (Eutheria: Carnivora) and tammar wallabies (Marsupialia: Diprotodontia) suggests that MsX and MSX genes serve as a common molecular link in unrelated diapausing mammals [12] (Figure 2). Mice are unable to maintain uterine quiescence and embryo survival under delayed conditions in the absence of uterine Msx [12]. These results suggest that the Msx genes are required 1) to coordinate uterine quiescence and blastocyst dormancy for survival during embryonic diapause, and 2) to maintain embryonic competency and uterine readiness for implantation.

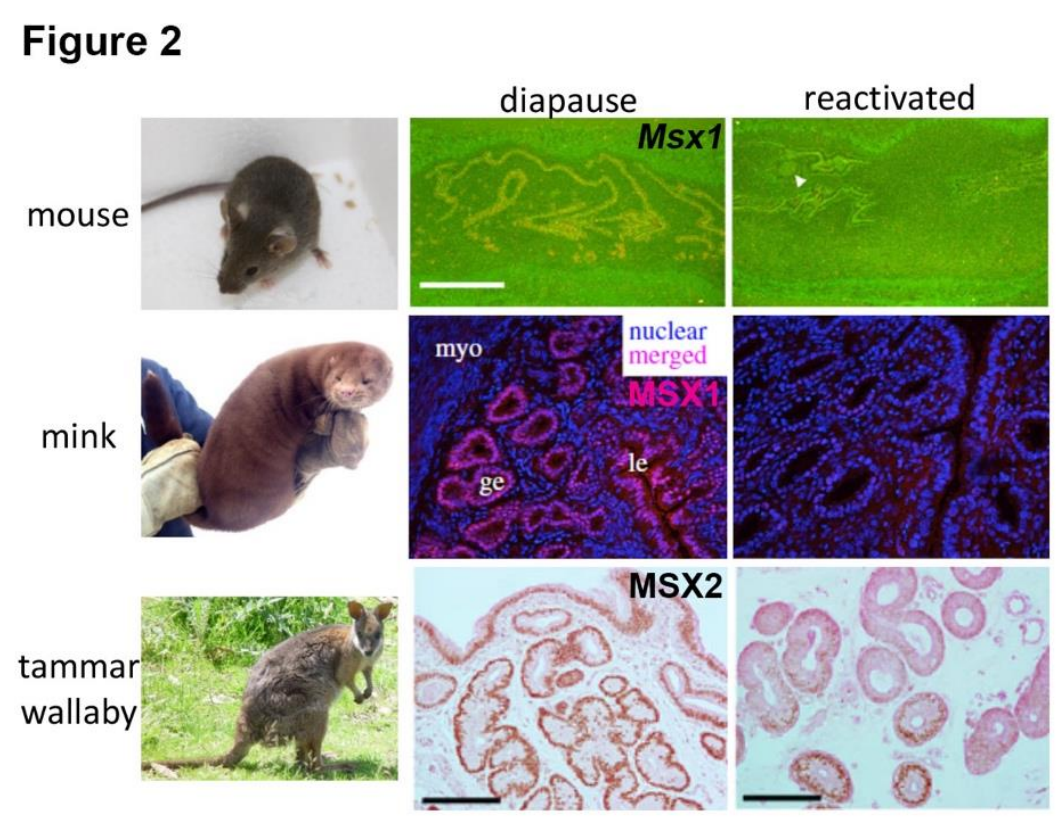

Figure 2. Proposed schematic of the regulation of diapause in mouse, mink and wallaby.

Top panels: ovariectomized, delayed implanting mice treated with progesterone or reactivated with progesterone and oestradiol-17ß were killed on day 8 of pregnancy. In situ hybridization showed distinct Msx1 expression in luminal and glandular epithelia during diapause with rapid downregulation following attachment reaction and embryonic activation $24 \mathrm{~h}$ after an oestradiol-17ß injection. Scale bar (mouse), 500 $\mu \mathrm{m}$. Arrowhead, blastocyst. Middle panels: immunofluorescence in the mink shows MSX1 nuclear localization during diapause with undetectable signal at the implantation site. Myo, myometrium; ge, glandular epithelium; le, luminal epithelium. Bottom panels: immunohistochemistry in the wallaby showed distinct nuclear localization of MSX2 in glandular and surface epithelia during diapause with gradual reduction on day 9 of reactivation from the surface epithelium and underlying glandular epithelium, but expression persisted in the deeper glands. Scale bar (mink and wallaby), $100 \mu \mathrm{m}$. 
These findings have opened up new avenues of research into examining the extent to which the molecular control of diapause, and indeed mammalian embryonic development in general, is evolutionarily conserved. This is the first evidence that experimentally manipulating the uterine environment can impact diapause in multiple species. The expression of a number of factors have now been examined across the mouse, mink and wallaby in attempts to study conservation of local mechanisms. Indeed, inhibition of the rate limiting enzyme of polyamine synthesis, ODC1, has been shown to be required for embryos to enter into diapause in both the mink and mouse $[18,19]$. Furthermore, DFMO treatment, an inhibitor of ODC-1, in mink results in the maintenance of uterine MSX1 expression [19].

It remains to be seen whether MSX genes are required for embryonic diapause in other species. Similar downregulation of MSX genes in early pregnancy has been reported in cattle, pig, and horse $[20,21]$. Whether human and non-human primates have, or once had, the potential to undergo delay remains unknown [1, 22]. Notably, gene array have shown that MSX1 and MSX2 are expressed in the human endometrium in a similar manner to that in mice, with higher expression prior to and downregulation during the window of implantation (mid-secretory phase). In addition, women with recurrent implantation failure were more likely to show dysfunctional MSX expression [23, 24]. Thus, we propose that MSX genes comprise a family of maternal regulators highly conserved not only for normal female fertility but also for the local regulation of mammalian embryonic diapause. We hope that these results may prompt investigators to explore the status of uterine MSX expression in other diapausing and non-diapausing species to expand our understanding of this conserved reproductive strategy and serve as a basis for interventions in animal conservation, fertility, and contraception.

\section{Declaration of interest}

The authors declare that there is no conflict of interest that could be perceived as prejudicing the impartiality of this article.

\section{Funding}

This work was supported in part by NIH grants (nos. HD12304, HD068524 and DA06668 to S.K.D.), Australian Research Council grants (to G.S. and M.B.R.) and a NSERC Canada grant (no. 137013 to B.D.M). J.C. was supported by an NIH NRSA Fellowship F30AG040858 and is now supported by NIH T32DK007061.

\section{References}

[1] Renfree MB and Fenelon JC. 2017 The enigma of embryonic diapause. Development 144(18) 31993210.

[2] Fenelon JC, Banerjee A and Murphy BD. 2014 Embryonic diapause: development on hold. Int J Dev Biol 58(2-4) 163-74.

[3] Mead RA. 1993 Embryonic diapause in vertebrates. J Exp Zool 266(6) 629-41.

[4] Yoshinaga K. 2013 A sequence of events in the uterus prior to implantation in the mouse. J Assist Reprod Genet 30(8) 1017-22.

[5] McLaren A. 1968 A study of blastocysts during delay and subsequent implantation in lactating mice. J Endocrinol 42(3) 453-63.

[6] Mantalenakis SJ and Ketchel MM. 1966 Frequency and extent of delayed implantation in lactating rats and mice. Journal of Reproduction and Fertility 12 391-394. 
[7] Weitlauf HM and Greenwald GS. 1968 Survival of blastocysts in the uteri of ovariectomized mice. J Reprod Fertil 17(3) 515-20.

[8] Ma WG et al. 2003 Estrogen is a critical determinant that specifies the duration of the window of uterine receptivity for implantation. Proc Natl Acad Sci USA 100(5) 2963-8.

[9] Cha J and Dey SK. 2014 Cadence of procreation: orchestrating embryo-uterine interactions. Semin Cell Dev Biol 34 56-64.

[10] Tyndale-Biscoe CHR, Renfree MB. 1987 Reproductive physiology of marsupials. Cambridge, UK: Cambridge University Press.

[11] Fenelon JC and Renfree MB. 2018 The history of the discovery of embryonic diapause in mammals. Biol Reprod 99(1) 242-251.

[12] Cha J et al. $2013 \mathrm{~A}$ new role for muscle segment homeobox genes in mammalian embryonic diapause. Open Biol 3(4) 130035.

[13] Finnerty JR, Mazza ME and Jezewski PA. 2009 Domain duplication, divergence, and loss events in vertebrate Msx paralogs reveal phylogenomically informed disease markers. BMC Evol Biol 918.

[14] Bendall AJ and Abate-Shen C. 2000 Roles for Msx and Dlx homeoproteins in vertebrate development. Gene 247(1-2) 17-31.

[15] Daikoku T et al. 2011 Conditional deletion of Msx homeobox genes in the uterus inhibits blastocyst implantation by altering uterine receptivity. Dev Cell 21(6) 1014-25.

[16] Fenelon JC et al. 2016 Polyamine-Mediated Effects of Prolactin Dictate Emergence from Mink Obligate Embryonic Diapause. Biol Reprod 95(1) 6.

[17] Cha J et al. 2015 Muscle Segment Homeobox Genes Direct Embryonic Diapause by Limiting Inflammation in the Uterus. J Biol Chem 290(24) 15337-49.

[18] Lefevre PL et al. 2011 Polyamines are implicated in the emergence of the embryo from obligate diapause. Endocrinology 152(4) 1627-39.

[19] Fenelon JC and Murphy BD. 2017 Inhibition of polyamine synthesis causes entry of the mouse blastocyst into embryonic diapause. Biol Reprod 97(1) 119-132.

[20] Bauersachs S. and Wolf E. 2013 Immune aspects of embryo-maternal cross-talk in the bovine uterus. J Reprod Immunol 97(1) 20-6.

[21] Samborski A et al. 2013 Deep sequencing of the porcine endometrial transcriptome on day 14 of pregnancy. Biol Reprod 88(4) 84.

[22] Ptak GE et al. 2012 Embryonic diapause is conserved across mammals. PLoS One 7(3) e33027.

[23] Riesewijk A et al. 2003 Gene expression profiling of human endometrial receptivity on days LH+2 versus LH+7 by microarray technology. Mol Hum Reprod 9(5) 253-64.

[24] Bolnick AD et al. 2016 Reduced homeobox protein MSX1 in human endometrial tissue is linked to infertility. Hum Reprod 31(9) 2042-50.

\section{Audience Discussion}

Colin Stewart: So, you didn't look at LIF receptor or GP130 expression?

Jeeyeon Cha: Those were unchanged.

GrazynaPtak: do you know anything more about the further development or the regression of these implantation sites?

Jeeyeon Cha: They are resorbed: we gave exogenous progesterone for the duration of day 20 and they still resorb.

Bruce Murphy: I think we know a lot about the temporal regulation about implantation, but we know very little about the spatial recognition. 
Jeeyeon Cha: That is one of the reasons why we considered WNT5A to be of importance in these animals because WNT5A is important for planar cell polarity. so this is a kind of spatial polarity where spacing is important: it is important for limb development or wing development in animals. It was first identified in feather development where spacing is very important. WNT5A knock-out mice show clustering of embryos and an inability to space evenly through the uterine horns as well and we found that to be true in WNT5A and its receptor RoR, and these result in resorption sites. There is something about the embryo spacing which is very important for embryo development.

Susanne Ulbrich: I have a question regarding the source of estrogen for the estrogen rise. You said that ovariectomy causes the loss of this estrogen rise, do you know whether this is of luteal or follicular origin?

Jeeyeon Cha: I assumed it was of follicular origin.

Susanne Ulbrich: Is this known?

Bruce Murphy: I don 't think this is known. It is a mystery. 